\title{
Integrating transcriptome-wide study and mRNA expression profiles yields novel insights into the biological mechanism of chondropathies
}

Ping Li ${ }^{\dagger}$, Yujie Ning ${ }^{\dagger}$, Xiong Guo, Yan Wen, Bolun Cheng, Mei Ma, Lu Zhang, Shiqiang Cheng, Sen Wang and Feng Zhang*

\begin{abstract}
Background: Chondropathies are a group of cartilage diseases, which share some common pathogenetic features. The etiology of chondropathies is still largely obscure now.

Methods: A transcriptome-wide association study (TWAS) was performed using the UK Biobank genome-wide association study (GWAS) data of chondropathies (including 1314 chondropathy patients and 450,950 controls) with gene expression references of muscle skeleton (MS) and peripheral blood (YBL). The candidate genes identified by TWAS were further compared with three gene expression profiles of osteoarthritis $(\mathrm{OA})$, cartilage tumor (CT), and spinal disc herniation (SDH), to confirm the functional relevance between the chondropathies and the candidate genes identified by TWAS. Functional mapping and annotation (FUMA) was used for the gene ontology enrichment analyses. Immunohistochemistry $(\mathrm{IHC})$ was conducted to validate the accuracy of integrative analysis results.

Results: Integrating TWAS and mRNA expression profiles detected 84 candidate genes for knee OA, such as DDX20 $\left(P_{\text {TWAS YBL }}=1.79 \times 10^{-3}\right.$, fold change $\left.(F C)=2.69\right)$, 10 candidate genes for $C$, such as SRGN $\left(P_{\text {TWAS YBL }}=1.46 \times 10^{-3}\right.$, $\mathrm{FC}=3.36)$, and 4 candidate genes for SDH, such as SUPV3L1 ( $\left.P_{\mathrm{TWAS} Y B L}=3.59 \times 10^{-3}, \mathrm{FC}=3.22\right)$. Gene set enrichment analysis detected $73 \mathrm{GO}$ terms for knee $\mathrm{OA}, 3 \mathrm{GO}$ terms for $\mathrm{CT}$, and $1 \mathrm{GO}$ term for $\mathrm{SDH}$, such as mitochondrial protein complex $\left(P=7.31 \times 10^{-5}\right)$ for knee OA, cytokine for $C T\left(P=1.13 \times 10^{-4}\right)$, and ion binding for SDH $\left(P=3.55 \times 10^{-4}\right)$. IHC confirmed that the protein expression level of DDX20 was significantly different between knee OA cartilage and healthy control cartilage $(P=0.0358)$.

Conclusions: Multiple candidate genes and $G O$ terms were detected for chondropathies. Our findings may provide a novel insight in the molecular mechanisms of chondropathies.
\end{abstract}

Keywords: Chondropathies, Genome-wide association studies (GWAS), Transcriptome-wide association study (TWAS), mRNA expression profile

\footnotetext{
*Correspondence: fzhxjtu@mail.xjtu.edu.cn

${ }^{\dagger}$ Ping Li and Yujie Ning contributed equally to this work.

Key Laboratory of Trace Elements and Endemic Diseases of National Health and Family Planning Commission, Key Laboratory of Environment and Genes Related to Diseases of Ministry of Education, Collaborative Innovation Center of Endemic Diseases and Health Promotion in Silk Road Region, School of Public Health, Health Science Center, Xi'an Jiaotong University, No.76 Yan Ta West Road, Xi'an 710061, Shaanxi, People's Republic of China
}

(c) The Author(s). 2019 Open Access This article is distributed under the terms of the Creative Commons Attribution 4.0 International License (http://creativecommons.org/licenses/by/4.0/), which permits unrestricted use, distribution, and reproduction in any medium, provided you give appropriate credit to the original author(s) and the source, provide a link to the Creative Commons license, and indicate if changes were made. The Creative Commons Public Domain Dedication waiver (http://creativecommons.org/publicdomain/zero/1.0/) applies to the data made available in this article, unless otherwise stated. 


\section{Introduction}

Chondropathies are a group of cartilage diseases that deviate from or interrupt the normal structure and function of cartilage, including osteoarthritis (OA), achondroplasia, spinal disc herniation $(\mathrm{SDH})$, relapsing polychondritis, cartilage tumor (CT), and chondrocalcinosis [1]. OA is the most well-known chondropathy in the world, affecting the health of approximately $15 \%$ of the population, especially people older than 60 years [2]. The prevalence of disc herniation, counting in the people who suffer from low back pain, is reported variable, approximately from 0 to $47 \%$ [3]. The biological mechanism of chondropathies remains largely unknown, and there is no effective way to treat the cartilage damage recently.

Risk factors for chondropathies mainly include trauma, genetics, age, sex, obesity, and degenerative pathology [2]. Recent studies demonstrated the implication of genetic factors in the development of chondropathies. For example, GDF5 was identified as a bone morphogenetic protein involved in joint formation [4]. The mice experiment has demonstrated the cooperation of Gli2 and p53 genes involved in the regulation of chondrocyte apoptosis in malignant cartilage tumors [5]. In addition, family and twin studies have detected that a large amount of genetic factors may be responsible for sciatica, disc herniation, and disc degeneration, and two collagen IX alleles have been identified as significant loci for sciatica and lumbar disc herniation [6].

Due to the non-vascular form, damaged cartilage has limited intrinsic capacity to repair and usually leads to tissue deterioration [7]. Another character of cartilage is its non-innervated form, which means cartilage damage shows asymptomatic in the early stage and becomes symptomatic with pain until it involves the adjacent innervated tissue [8]. These are the common processes shared by various chondropathies. Moreover, cartilage matrix degradation, chondrocyte death, inflammation, and mitochondrial dysfunction have been demonstrated as the homologous features in more than two types of chondropathies. For instance, inflammation is involved in $\mathrm{OA}$, relapsing polychondritis, and $\mathrm{SDH}$. Cartilage matrix degradation could be found in most types of chondropathies [9]. Moreover, some common causal genes have been demonstrated in different types of chondropathies, such as tumor necrosis factor $\alpha$ (TNF$\alpha)$ and interleukin $1 \beta$ (IL-1 $\beta$ ) [10]. However, limited efforts have been made to explore the common biological mechanism of various chondropathies. Most previous studies only focused on one type of chondropathies, such as knee OA, hip OA, CT, or SDH, which masked the homologous features for all the chondropathies.

Over the past few years, genome-wide association studies (GWAS) have identified significant genetic variants for chondropathies [11]. However, GWAS-identified variants are located in non-coding regions, which could hardly explain the relative risk [12]. The genes contributing to these associations remain largely unknown. Recently, integrative analysis of GWAS and noncoding genetic regulatory loci information has attracted more attention. A powerful approach called transcriptome-wide association study (TWAS) was proposed to identify expression-trait based causal genes by integrating gene expression data with GWAS data [13]. The TWAS, based on the GWAS data and gene expression imputation, shows effectively in identifying the causal genes whose expression are related to human complex traits and diseases [12].

In this study, TWAS was adopted from a large-scale GWAS of chondropathies to identify mRNA expression associated genes for chondropathies. Next, the candidate genes identified by TWAS were compared with the mRNA expression profiles of $\mathrm{OA}, \mathrm{CT}$, and $\mathrm{SDH}$, to further confirm the functional relevance of identified candidate genes with chondropathies. The overlapped common genes shared by TWAS and mRNA expression profiles were subjected to functional enrichment analyses.

\section{Methods}

\section{The GWAS summary data}

The GWAS summary data of chondropathies were obtained from the UK Biobank (http://geneatlas.roslin.ed. ac.uk/). All study subjects were of European ancestry, including 1314 chondropathy patients and 450,950 controls, aged between 40 and 69 years (UK Biobank Field: $41202,41204)[14,15]$. The UK Biobank is a large prospective epidemiological study, recruiting nearly 500,000 deeply phenotyped individuals [14]. Briefly, DNA was extracted from blood samples stored at the UK Biobank facility for genotyping. Both marker-based quality control and sample-based quality control procedures were implemented by principal component analysis accounting for possible population structure in the study samples [14]. Genome-wide genotyping was conducted using either the Affymetrix UK BiLEVE Axiom or Affymetrix UK Biobank Axiom array. Linear mixed model was used for genome-wide SNP association testing. Detailed information of study samples, study design, and statistical analysis could be found in the published study $[14,15]$.

\section{Gene expression profiles of knee OA}

The mRNA expression profiles of knee OA cartilage tissue were obtained from a previous study [16]. The knee cartilage specimens were collected from 6 knee OA patients ( 1 male/5 females) and 5 normal control ( 3 males/ 2 females). Each cartilage specimen was disserted into two groups, one for microarray experiment and the 
other for quantitative reverse transcription polymerase chain reaction (qRT-PCR) validation. The extracted mRNA was purified, amplified, and transcribed into fluorescent cRNA after removing rRNA from the total RNA. The quality of cRNAs was measured using the Nanodrop ND-1000. Then, cRNA was hybridized to the Human lncRNA Array following the Agilent One-Color Microarray-Based Gene Expression Analysis protocol (Agilent Technology). Significant regulated mRNAs were defined as fold change (FC) $\geq 2.0$ and $P$ value $<0.05$. Detailed information of study samples, study design, and statistical analysis could be found in Additional file 1: Table S1 and the published study [16].

\section{Gene expression profiles of $\mathrm{CT}$}

The mRNA expression profiles of CT were obtained from the GEO database (https://www.ncbi.nlm.nih.gov/ geo/) (Access number: GSE22855) [17]. Ollier disease is a rare disorder and develops multiple benign cartilage tumors called enchondromas [17]. This dataset contained 7 Ollier enchondrama patients as well as 2 growth plates and 4 articular cartilage served as controls [17]. Total RNA was isolated from the fresh frozen tissue and measured by an RNA 600 Nano LabChip kit with Agilent 2100 Bioanalyzer (Santa Clara, CA, USA). Genome-wide mRNA expression profiling was conducted using the Illumina BeadArray v3.0 Chip. The processed expression profile data was analyzed by the linear models for microarray (LIMMA) tool. Detailed information of study samples, study design, and statistical analysis could be found in Additional file 1: Table S1 and the published study [17].

\section{Gene expression profiles of SDH}

The significantly differently expressed genes in SDH were obtained from a published study [18]. Briefly, intervertebral disc (IVD) tissue specimens were collected from 10 degenerative disc patients $(6$ females $/ 4$ males, $21-43$ years) and 10 healthy controls ( 5 females $/ 5$ males, 28-55 years). Total RNA was isolated from whole IVD tissue, reverse-transcribed into cRNA, and hybridized to Agilent Human 1A microarray chip. Microarrays were scanned by Gene-Pix 4000B and analyzed by GenePixPro 3.0 (Axon Instruments, Inc., CA, USA). The genes were considered significantly differentially expressed when $P$ value $<0.05$ and $\mathrm{FC} \geq 2.0$. Detailed information of study samples, study design, and statistical analysis could be found in Additional file 1: Table S1 and the published study [18].

\section{Immunohistochemistry (IHC)}

Two genes (CSF1R and DDX20) of the top 10 candidate genes of knee OA were randomly selected for IHC. Knee cartilage specimens were collected from 5 knee OA patients and 4 heathy controls (Additional file 2: Table S2). Healthy knee specimens were collected from the subjects undergoing amputation caused by traffic accident. Primary knee OA was diagnosed according to careful clinical and radiography examination. Subjects with genetic bone, cartilage, and other skeletal disorders were excluded from this study. For IHC, the cartilage specimens were prepared and embedded in paraffin. Then, deparaffinization and rehydration by citrate buffer $(\mathrm{pH} 6.0)$ overnight at $37^{\circ} \mathrm{C}$ and $12.5 \%$ trypsin (Xi'an GuoAn Biological Technology Co.) at $37^{\circ} \mathrm{C}$ for $20-30$ min were done. After that, Zhong Shan Gold Bridge Rabbit SP reagent was used in the following procession (Beijing Zhong Gold Bridge Biological Technology Co., 18112A11) according to the manual: $3 \%$ hydrogen peroxide solution for $10 \mathrm{~min}$ at room temperature (RT) (white bottle), blocking in serum for 20 min at RT (blue bottle), primary antibody of CSF1R (1:50, Proteintech) and DDX20 (1:50, Proteintech) incubated overnight at $4{ }^{\circ} \mathrm{C}$, secondary antibody for $18 \mathrm{~min}$ at $37^{\circ} \mathrm{C}$ (yellow bottle), and horseradish peroxidase (HRP) for 18 min at $37^{\circ} \mathrm{C}$ (red bottle).

\section{Statistical analysis}

The TWAS of chondropathies was performed by the FUSION software (http://gusevlab.org/projects/fusion/) [13]. Using pre-computed gene expression weights together with GWAS summary data, FUSION is capable of evaluating the gene expression associations between every gene and target diseases. In this work, TWAS analysis was performed based on the chondropathies GWAS dataset obtained from the UK Biobank as well as gene expression references of muscle skeleton (MS) and peripheral blood (YBL) from FUSION [13, 15]. For mRNA expression profile analysis, the differentially expressed genes of knee OA and SDH were identified using an unpaired $t$-test. The mRNA expression profile analysis of CT (Ollier disease) was performed using the GEO2R tool of GEO [19]. The candidate genes identified by TWAS were compared with the significant expressed genes detected by mRNA expression profiles to detect common genes. GO enrichment analyses of the identified common genes was performed by the functional mapping and annotation (FUMA) software [20]. For IHC data analysis, ImageJ was used to analyze the percentage of positive chondrocytes and $t$-test was conducted using SPSS 19.0.

\section{Results}

TWAS results of chondropathies

TWAS of chondropathies identified 195 genes in MS and 252 genes in YBL with $P_{\text {TWAS }}<0.05$ (Additional file 3: Table S3, and Additional file 4: Table S4). For instance, NSA2 $\left(P_{\text {TWAS }} \mathrm{MS}=2.47 \times 10^{-4}\right)$, TP53I13 $\left(P_{\text {TWAS }} \mathrm{MS}=\right.$ $\left.2.63 \times 10^{-4}\right)$, and FPGT $\left(P_{\text {TWAS MS }}=3.69 \times 10^{-4}\right)$ were 
the top three significant genes in MS, and RSRC1 ( $P_{\text {TWAS }}$ $\left.\mathrm{YBL}=1.03 \times 10^{-4}\right)$, NSA2 $\left(P_{\text {TWAS }} \mathrm{YBL}=3.30 \times 10^{-4}\right)$, and TPD52 $\left(P_{\text {TWAS }}\right.$ YBL $\left.=3.62 \times 10^{-4}\right)$ were the top three significant genes in YBL.

We also detected 20 overlapped genes in both MS and YBL (Table 1$)$ in chondropathies, such as NSA2 ( $P_{\text {TWAS }}$ $\left.\mathrm{MS}=2.47 \times 10^{-4}, \quad P_{\text {TWAS }} \quad \mathrm{YBL}=3.30 \times 10^{-4}\right), \quad$ RSRC1 $\left(P_{\text {TWAS }} \quad \mathrm{MS}=4.54 \times 10^{-4}, \quad P_{\mathrm{TWAS}} \quad \mathrm{YBL}=1.03 \times 10^{-4}\right)$, NME6 $\left(P_{\text {TWAS MS }}=9.93 \times 10^{-3}, P_{\text {TWAS YBL }}=1.75 \times 10^{-}\right.$ $\left.{ }^{3}\right)$, NUDT2 $\left(P_{\text {TWAS MS }}=1.85 \times 10^{-2}, P_{\text {TWAS YBL }}=2.32 \times\right.$ $\left.10^{-2}\right)$, and ACADM $\left(P_{\text {TWAS MS }}=3.03 \times 10^{-2}, P_{\text {TWAS }}\right.$ $\mathrm{YBL}=4.14 \times 10^{-2}$ ).

\section{Integrative analysis of TWAS and mRNA expression profiles}

The top 10 candidate genes identified for knee OA, CT, and SDH are listed in Table 2. For knee OA, we detected 84 overlapped genes shared by TWAS and mRNA expression profiling (Additional file 5: Table S5), such as NSA2 $\left(P_{\text {TWAS MS }}=2.47 \times 10^{-4}, P_{\text {TWAS YBL }}=3.30 \times 10^{-4}\right.$, mRNA $\mathrm{FC}=-2.16), \quad \operatorname{CSF} 1 \mathrm{R}\left(P_{\mathrm{TWAS}} \mathrm{YBL}=3.95 \times 10^{-4}\right.$, mRNA $\quad \mathrm{FC}=3.47), \quad \mathrm{MSC} \quad\left(P_{\mathrm{TWAS}} \mathrm{YBL}=9.51 \times 10^{-4}\right.$, mRNA FC $=4.41)$, CDK5R1 $\left(P_{\text {TWAS }}\right.$ YBL $=1.22 \times 10^{-3}$, mRNA FC $=2.24), \quad$ MMP24 $\left(P_{\text {TWAS }}\right.$ MS $=1.49 \times 10^{-3}$,

Table 1 List of 20 overlapped genes identified by TWAS in both MS and YBL for chondropathies

\begin{tabular}{|c|c|c|c|}
\hline Gene & Chromosome & $P_{\text {TWAS MS }}$ & $P_{\text {TWAS YBL }}$ \\
\hline$\overline{\text { ACADM }}$ & 1 & $3.03 \times 10^{-2}$ & $4.14 \times 10^{-2}$ \\
\hline ADIPOR1 & 1 & $3.83 \times 10^{-2}$ & $9.15 \times 10^{-3}$ \\
\hline AGA & 4 & $4.34 \times 10^{-2}$ & $1.48 \times 10^{-2}$ \\
\hline ARMC1 & 8 & $1.62 \times 10^{-3}$ & $8.76 \times 10^{-3}$ \\
\hline CHURC1 & 14 & $1.59 \times 10^{-2}$ & $1.63 \times 10^{-2}$ \\
\hline FAM149B1 & 10 & $5.03 \times 10^{-3}$ & $5.30 \times 10^{-3}$ \\
\hline FPGT & 1 & $3.69 \times 10^{-4}$ & $1.40 \times 10^{-2}$ \\
\hline KHK & 2 & $1.57 \times 10^{-2}$ & $1.02 \times 10^{-2}$ \\
\hline MTFR1 & 8 & $5.32 \times 10^{-3}$ & $3.30 \times 10^{-2}$ \\
\hline NDFIP1 & 5 & $7.48 \times 10^{-3}$ & $3.82 \times 10^{-2}$ \\
\hline NDUFA10 & 2 & $1.56 \times 10^{-2}$ & $6.11 \times 10^{-3}$ \\
\hline NME6 & 3 & $9.93 \times 10^{-3}$ & $1.75 \times 10^{-3}$ \\
\hline NSA2 & 5 & $2.47 \times 10^{-4}$ & $3.30 \times 10^{-4}$ \\
\hline NUDT13 & 10 & $9.46 \times 10^{-3}$ & $2.77 \times 10^{-3}$ \\
\hline NUDT2 & 9 & $1.85 \times 10^{-2}$ & $2.32 \times 10^{-2}$ \\
\hline PHLPP2 & 16 & $7.58 \times 10^{-3}$ & $1.88 \times 10^{-2}$ \\
\hline RSRC1 & 3 & $4.54 \times 10^{-4}$ & $1.03 \times 10^{-4}$ \\
\hline SH3GLB2 & 9 & $1.46 \times 10^{-2}$ & $7.00 \times 10^{-3}$ \\
\hline TAF1B & 2 & $2.85 \times 10^{-2}$ & $2.38 \times 10^{-2}$ \\
\hline TRIM66 & 11 & $2.80 \times 10^{-2}$ & $2.86 \times 10^{-2}$ \\
\hline
\end{tabular}

Note: $P_{\text {TWAS MS }}$ and $P_{\text {TWAS YBL }}$ denote the TWAS $P$ value in muscle skeleton (MS) and peripheral blood (YBL)
Table 2 The top 10 candidate genes detected by TWAS and mRNA expression profile analysis for knee OA, CT, and SDH

\begin{tabular}{|c|c|c|c|c|c|}
\hline \multirow[t]{2}{*}{ Chondropathies } & \multirow[t]{2}{*}{ Genes } & \multicolumn{2}{|l|}{ TWAS } & \multicolumn{2}{|c|}{ mRNA expression } \\
\hline & & Tissue & $P_{\text {TWAS }}$ & $\overline{F C}$ & $P_{\text {mRNA }}$ \\
\hline \multirow[t]{10}{*}{ Knee OA } & NSA2 & $M S / Y B L$ & $\begin{array}{l}2.47 \times 10^{-4} / \\
3.30 \times 10^{-4}\end{array}$ & -2.16 & $2.12 \times 10^{-2}$ \\
\hline & CSF1R & YBL & $3.95 \times 10^{-4}$ & 3.47 & $2.83 \times 10^{-2}$ \\
\hline & MSC & YBL & $9.51 \times 10^{-4}$ & 4.41 & $2.03 \times 10^{-2}$ \\
\hline & CDK5R1 & YBL & $1.22 \times 10^{-3}$ & 2.24 & $4.71 \times 10^{-2}$ \\
\hline & MMP24 & MS & $1.49 \times 10^{-3}$ & 2.41 & $2.64 \times 10^{-2}$ \\
\hline & ARMC1 & MS/ YBL & $\begin{array}{l}1.62 \times 10^{-3} / \\
8.76 \times 10^{-3}\end{array}$ & -2.64 & $4.43 \times 10^{-2}$ \\
\hline & NCK1 & YBL & $1.75 \times 10^{-3}$ & -2.26 & $4.73 \times 10^{-2}$ \\
\hline & NME6 & $\mathrm{MS} / \mathrm{YBL}$ & $\begin{array}{l}9.93 \times 10^{-3} / \\
1.75 \times 10^{-3}\end{array}$ & -2.93 & $2.22 \times 10^{-3}$ \\
\hline & DDX20 & YBL & $1.79 \times 10^{-3}$ & 2.69 & $1.15 \times 10^{-2}$ \\
\hline & SCARB1 & YBL & $2.55 \times 10^{-3}$ & -3.27 & $6.75 \times 10^{-3}$ \\
\hline \multirow[t]{10}{*}{$C T$} & SRGN & YBL & $1.46 \times 10^{-3}$ & 3.36 & $4.18 \times 10^{-2}$ \\
\hline & ETS1 & YBL & $3.07 \times 10^{-3}$ & -2.16 & $4.05 \times 10^{-5}$ \\
\hline & PFKFB3 & YBL & $4.28 \times 10^{-3}$ & 3.66 & $7.00 \times 10^{-3}$ \\
\hline & CHURC1 & MS/ YBL & $\begin{array}{l}1.59 \times 10^{-2} / \\
1.63 \times 10^{-2}\end{array}$ & -2.01 & $1.36 \times 10^{-2}$ \\
\hline & TRMT112 & YBL & $1.94 \times 10^{-2}$ & -2.56 & $1.01 \times 10^{-6}$ \\
\hline & PLEKHA1 & MS & $2.29 \times 10^{-2}$ & -2.62 & $7.66 \times 10^{-5}$ \\
\hline & AFF3 & YBL & $2.62 \times 10^{-2}$ & -2.35 & $2.08 \times 10^{-4}$ \\
\hline & $\mathrm{S} 100 \mathrm{P}$ & YBL & $3.31 \times 10^{-2}$ & 5.49 & $2.29 \times 10^{-2}$ \\
\hline & PSMC5 & YBL & $4.68 \times 10^{-2}$ & -2.22 & $4.21 \times 10^{-7}$ \\
\hline & C1QTNF4 & MS & $4.96 \times 10^{-2}$ & 3.32 & $2.38 \times 10^{-5}$ \\
\hline \multirow[t]{4}{*}{ SDH } & SUPV3L1 & YBL & $3.59 \times 10^{-3}$ & 3.22 & - \\
\hline & ZNF195 & YBL & $6.67 \times 10^{-3}$ & 2.25 & - \\
\hline & $\mathrm{HBG} 2$ & YBL & $2.36 \times 10^{-2}$ & 3.91 & - \\
\hline & VAMP4 & YBL & $4.77 \times 10^{-2}$ & 2.23 & - \\
\hline
\end{tabular}

Note: MS denotes muscle skeleton. YBL denotes peripheral blood. FC denotes mRNA expression fold change. TWAS means transcriptome-wide association. $P_{\text {TWAS }}$ means the $P$ value of TWAS. $P_{\text {mRNA }}$ means the $P$ value of mRNA expression. The $P_{\text {mRNA }}$ of the mRNA expression profile of SDH was not reported in the published study

mRNA FC $=2.41)$, and DDX20 $\left(P_{\text {TWAS YBL }}=1.79 \times 10^{-3}\right.$, mRNA FC $=2.69$ ).

For CT, 10 overlapped genes were shared by TWAS and mRNA expression profiling (Additional file 6: Table S6), such as SRGN $\left(P_{\text {TWAS }}\right.$ YBL $=1.46 \times 10^{-3}$, mRNA $\mathrm{FC}=3.36)$, ETS1 $\left(P_{\text {TWAS }}\right.$ YBL $=3.07 \times 10^{-3}$, mRNA FC $=$ - 2.16), PFKFB3 $\left(P_{\text {TWAS YBL }}=4.28 \times 10^{-3}\right.$, mRNA FC $=$ 3.66), CHURC1 $\left(P_{\text {TWAS MS }}=1.59 \times 10^{-2}, P_{\text {TWAS }} \mathrm{YBL}=\right.$ $1.62 \times 10^{-2}$, mRNA $\left.\mathrm{FC}=-2.01\right)$, and $\mathrm{S} 100 \mathrm{P}$ ( $P_{\text {TWAS }}$ $\left.\mathrm{YBL}=3.31 \times 10^{-2}, \mathrm{mRNA} F \mathrm{FC}=5.49\right)$.

For SDH, integrating TWAS and mRNA expression profiles detected four common genes (Additional file 7: Table S7), including SUPV3L1 $\left(P_{\text {TWAS YBL }}=3.59 \times 10^{-3}\right.$, mRNA FC $=3.22), \quad$ ZNF195 $\left(P_{\text {TWAS }}\right.$ YBL $=6.67 \times 10^{-3}$, 
mRNA $\quad \mathrm{FC}=2.25), \quad$ HBG2 $\quad\left(P_{\text {TWAS }} \quad \mathrm{YBL}=2.36 \times 10^{-2}\right.$, mRNA FC $=3.91)$, and VAMP4 $\left(P_{\text {TWAS YBL }}=4.77 \times 10^{-}\right.$ 2 , mRNA FC $=2.23$ ).

Interestingly, 4 common genes were found both in knee OA and CT, such as ETS1 $\left(P_{\text {TWAS YBL }}=3.07 \times 10^{-}\right.$ , mRNA $\mathrm{FC}_{\mathrm{OA}}=2.34$, mRNA $\mathrm{FC}_{\mathrm{CT}}=-2.16$ ), PFKFB3 $\left(P_{\text {TWAS YBL }}=4.28 \times 10^{-3}\right.$, mRNA $\mathrm{FC}_{\mathrm{OA}}=-4.46, \mathrm{mRNA}$ FC $\left._{\mathrm{CT}}=3.66\right)$, CHURC1 $\left(P_{\text {TWAS MS }}=1.59 \times 10^{-2}, P_{\text {TWAS }}\right.$ $\mathrm{YBL}=1.63 \times 10^{-2}$, mRNA $\mathrm{FC}_{\mathrm{OA}}=-5.32$, mRNA $\mathrm{FC}_{\mathrm{CT}}=$ -2.01 ), and C1QTNF4 $\left(P_{\text {TWAS MS }}=4.96 \times 10^{-2}\right.$, mRNA $\mathrm{FC}_{\mathrm{OA}}=-4.82$, mRNA $\mathrm{FC}_{\mathrm{CT}}=3.32$ ).

\section{Gene ontology (GO) enrichment analyses}

The identified common genes shared by TWAS and mRNA expression profiles were subjected to GO enrichment analysis. For knee OA, we identified $73 \mathrm{GO}$ terms (Table 3, Additional file 8: Table S8), such as mitochondrial protein complex $\left(P=7.31 \times 10^{-5}\right)$, mitochondrial membrane part $\left(P=1.81 \times 10^{-4}\right)$, oxidoreductase complex $\left(P=4.87 \times 10^{-4}\right)$, and regulation of lipid catabolic process $\left(P=4.40 \times 10^{-4}\right)$. For CT, $3 \mathrm{GO}$ terms were detected, including response to tumor necrosis factor $(P=$ $\left.9.22 \times 10^{-5}\right)$, response to cytokine $\left(P=1.13 \times 10^{-4}\right)$, and positive regulation of defense response $\left(P=3.46 \times 10^{-4}\right)$. The transition metal ion binding $\left(P=3.55 \times 10^{-4}\right)$ was the only GO term detected for SDH.

\section{IHC validation}

The expression levels of CSF1R and DDX20 were evaluated in the cartilage specimens from knee OA patients and healthy controls (Figs. 1 and 2). IHC results showed that the percentage of positive chondrocytes of DDX20 in knee OA cartilage (0.44\%) was significantly higher compared to heathy cartilage $(0.06 \%, P=0.0358)$. CSF1R also showed overexpression in knee OA cartilage compared to heathy controls $(0.25 \%$ vs. $0.11 \%)$, but not statistically significant $(P=0.5304)$.

\section{Discussion}

Recently, GWAS have successfully identified amount of candidate variants for complex diseases. However, the identified loci are almost located at non-coding regions and could hardly explain the relative risk [12]. Therefore, in order to yield an insight on the mechanism of chondropathies, we performed TWAS by using large-scale GWAS data of chondropathies. Then, the results of TWAS were integrated with different mRNA expression data of three types of chondropathies to find causal genes for knee OA, CT, and SDH.

Integrative TWAS and mRNA expression profiles detected multiple candidate genes for knee OA, such as DDX20 and NSA2. DDX20, a member of the Asp-GluAla-Asp (DEAD) box protein family, a component of microRNA-containing ribonucleoprotein complexes, encodes an RNA helicase. Previous study suggested that miRNA-140 plays a central role in DDX20 deficiency-related pathogenesis, taking part in the classical nuclear factor- $\mathrm{kB}(\mathrm{NF}-\mathrm{kB})$ pathway and targeting DNA metyltransferase 1 expression [21]. Recently, the NF- $\mathrm{kB}$ pathway has been found to regulate chondrocyte proliferation and differentiation and maintain endochondral ossification [22]. NSA2 was the most significant gene identified by TWAS for chondropathies, also detected by the mRNA expression profiling of knee OA cartilage. NSA2 encodes Nop seven-associated 2 ribosome biogenesis factor. It is a nucleolar protein required for ribosome biogenesis and involved in cell cycle regulation and proliferation [23]. Recently, NSA2 has been reported as the candidate gene for diabetic nephropathy and is related to the TGF- $\beta 1$ pathway [24].

Besides, we have identified several significant genes associated with CT, such as SRGN. Its official full name is serglycin. The protein of this gene is best known as a hematopoietic cell granule proteoglycan, which may be vital for neutralizing hydrolytic enzymes and involved in the granule-mediated apoptosis. SRGN is over expressed

Table 3 The top 10 gene ontology terms identified for knee OA

\begin{tabular}{llll}
\hline Gene ontology & Tissue & $P$ value & Adjusted $P$ value \\
\hline GO_MATURATION_OF_LSU_RRNA & MS & $1.18 \times 10^{-6}$ & $5.21 \times 10^{-3}$ \\
GO_NADH_DEHYDROGENASE_COMPLEX & MS & $4.56 \times 10^{-5}$ & $1.84 \times 10^{-2}$ \\
GO_MITOCHONDRIAL_PROTEIN_COMPLEX & MS & $7.31 \times 10^{-5}$ & $1.84 \times 10^{-2}$ \\
GO_MITOCHONDRIAL_MEMBRANE_PART & MS & $1.81 \times 10^{-4}$ & $2.10 \times 10^{-2}$ \\
GO_RESPIRATORY_CHAIN & MS & $2.90 \times 10^{-4}$ & $2.81 \times 10^{-2}$ \\
GO_OXIDOREDUCTASE_COMPLEX & MS & $4.87 \times 10^{-4}$ & $3.18 \times 10^{-2}$ \\
GO_INNER_MITOCHONDRIAL_MEMBRANE_PROTEIN_COMPLEX & MS & $7.14 \times 10^{-4}$ & $3.45 \times 10^{-2}$ \\
GO_PURINE_NUCLEOBASE_BIOSYNTHETIC_PROCESS & YBL & $4.75 \times 10^{-6}$ & $2.11 \times 10^{-2}$ \\
GO_NUCLEOBASE_BIOSYNTHETIC_PROCESS & YBL & $1.74 \times 10^{-5}$ & $3.86 \times 10^{-2}$ \\
GO_PURINE_NUCLEOBASE_METABOLIC_PROCESS & YBL & $2.82 \times 10^{-5}$ & $4.17 \times 10^{-2}$ \\
\hline
\end{tabular}

Note: MS denotes muscle skeleton. YBL denotes peripheral blood 

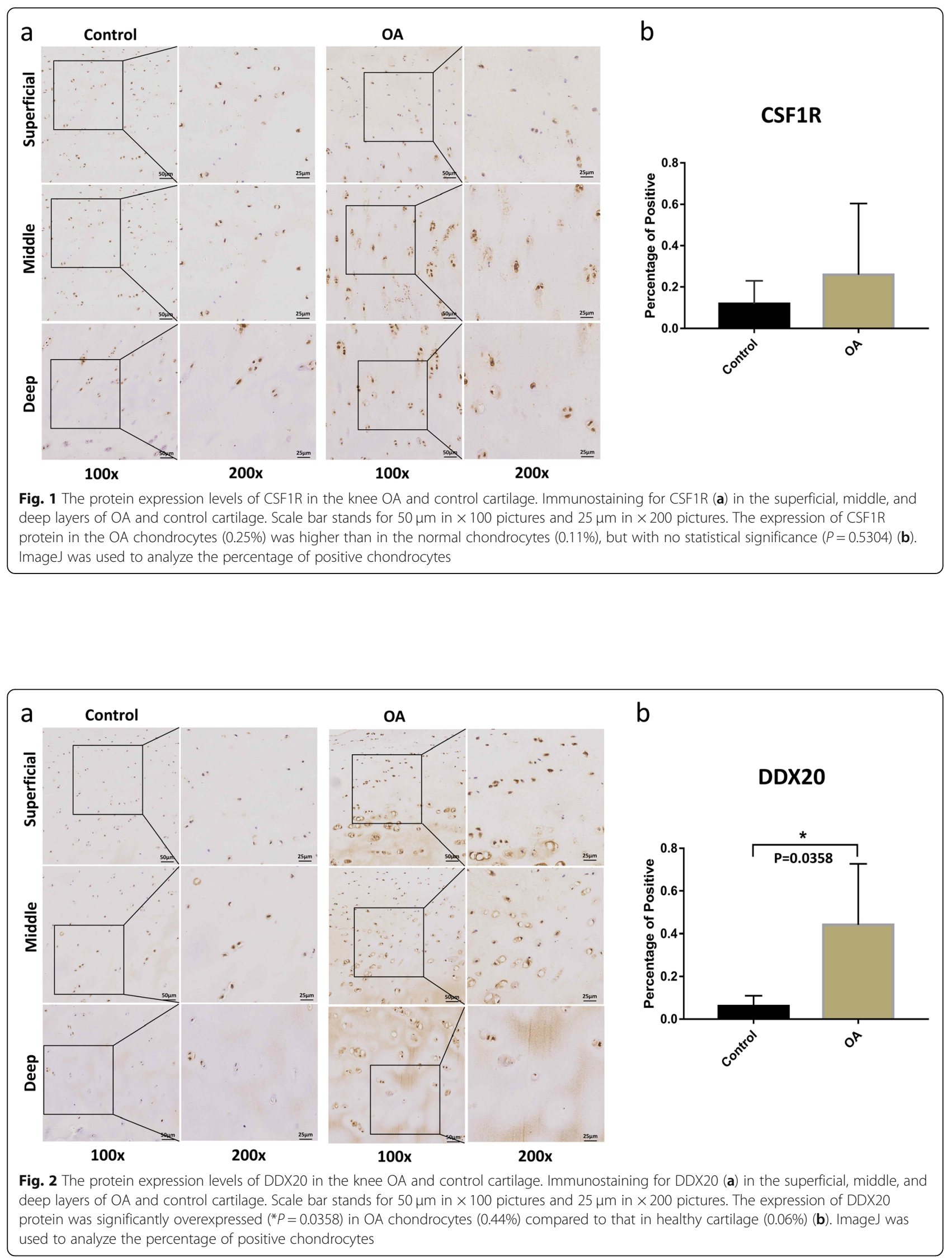
in tumor cells and associated with tumor cell aggressiveness and poor prognosis in cancers [25]. Another study detected SRGN is markedly synthesized by cancer and stromal cells in malignant cancers [26], indicating a critical role of SRGN in the procession of cancer.

Another notable gene for CT is S100P, which is a member of the S100 family of proteins containing 2 EFhand calcium-binding motifs. The protein encoded by this gene could not only bind $\mathrm{Ca} 2+, \mathrm{Zn} 2+$, and $\mathrm{Mg} 2+$, but also play an important role in the progress of various tumors [27]. Yang et al. have illustrated the relationship between blood-based S100P methylation and breast cancer [28]. Moreover, Piltti et al. demonstrated that S100P was the only significantly changed S100 protein family member and its expression was 2.5 times higher than the control in human chondrosarcoma HCS-2/8 cells [29]. This result is almost coincident with our findings about S100P, showing that S100P was overexpressed in $\mathrm{CT}$ and may be a causal gene for the disease.

Interestingly, we also found 4 common genes both in knee OA and CT, such as PFKFB3. It belongs to a family of bifunctional proteins involved in both the synthesis and degradation of fructose-2,6-bisphosphate, and in charge of glycolysis in eukaryotes. A previous study has demonstrated the important role of PFKFB3 in regulating the glycolytic metabolism in human OA cartilage [30]. Besides, PFKFB3 is also identified in glycolysis, cell proliferation, and tumor growth in the procession of tumor and inflammation [31]. Taken together, it would be of significant importance to further detect the role of PFKFB3 involved in the development of knee OA and $\mathrm{CT}$, and the potential treatment target of PFKFB3 for both of them.

For SDH, we detected 4 causal genes. For instance, ZNF195, zinc finger protein 195, located at chromosome $11 \mathrm{p} 15.5$, is a member of the Krueppel C2H2-type zincfinger protein family. The family mainly includes transcription factors and participants in various cellular processes. Previous studies have shown that ZNF195 is related to various cancers, such as cutaneous $\mathrm{T}$ cell lymphoma [32]. The expression of ZNF195 was closely related to gemcitabine sensitivity in head and neck squamous cell carcinoma (HNSCC) and decreased in the low level of oxygen in first trimester human trophoblast cells [33]. However, few researches have demonstrated the function of ZNF195, and none about this gene in SDH. Further study is needed to identify the mechanism of ZNF195 involved in the development and progression of $\mathrm{SDH}$.

Multiple GO terms were enriched in the candidate genes of the three types of chondropathies. For knee $\mathrm{OA}$, mitochondria were identified as the major parts of GO cellular component terms, such as mitochondrial membrane part (GO:0044455), inner mitochondrial membrane protein complex (GO:009800), and mitochondrial protein complex (GO:0098798). It has been identified that mitochondria are important regulators of cellular function and survival and play a vital role in aging-related diseases, especially in OA [34]. Our results further confirmed the importance of mitochondria and highlighted the mitochondrial membrane in the etiology of knee OA. In addition, the association of oxidoreductase (GO:1990204, GO:0034614, GO:0055114, GO: 0016491) and lipid (GO:0050994, GO:0046890, GO: 0046889) GO terms was found as the main biological process and molecular function terms changed in knee OA. Poloma et al. [35] have demonstrated that decreasing mitochondrial membrane potential is related to increasing reactive oxygen species (ROS) production and cell death in OA. Tang et al. [36] indicated that reversing oxidative stress-mediated mitochondrial membrane potential collapse could be an effective way for OA protection against mitochondrial dysfunction. On the other hand, obesity is a major risk factor for OA. The dyslipidemia plays an important role in obesity-induced OA [37]. Lipid deposition in the joint was detected at the early stages of OA before histological changes [38]. Multiple lipid metabolism-related genes have been identified dramatically reduced in OA articular cartilage compared to normal samples, such as ATP-binding-cassette transporter A1 (ABCA1), apoliproprotein A1 (ApoA1), liver $X$ receptor alpha (LXR $\alpha$ ), and liver $X$ receptor beta (LXR $\beta$ ) [39]. Therefore, the function of mitochondria, oxidoreductase, and lipid could not be underestimated in the OA development.

For $\mathrm{CT}$, we detected that the biological processes of $\mathrm{CT}$ are mainly related to tumor necrosis factor (GO: 0034612), cytokine (GO:0034097), and positive regulation of defense response (GO:0031349). Many studies have shown that TNF as well as cytokine, such as IL-1, IL-6, and IL-17, has been involved in the cartilage degradation [40]. It has been identified that TNF regulates the inflammatory cascade, and IL- $1 \beta$ is related to cartilage destruction. TNF and IL-1 $\beta$ play a critical role in the cartilage degradation of $\mathrm{OA}$ [41]. The findings in our study may give a novel molecular mechanism insight in CT procession.

For SDH, only one GO molecular function term was significantly enriched, which is transition metal ion binding (GO:0046914). There are eight metals involved in the biologically relevant transition metals, including vanadium, manganese, iron, copper, cobalt, nickel, molybdenum, and silver. Copper deficiency has been identified as a critical factor in antioxidant defenses, mitochondrial energy production, and in iron metabolism in blood and muscles in myeloneuropathy [42]. Therefore, it would be worthy to deeply evaluate the other transition metals in SDH procession. 
There are two innovations in our study. First, given the common biological processes shared by different types of chondropathies, we conducted a TWAS using chondropathies as phenotypes. This approach may help us to detect novel susceptibility genes contributing to the overlapped genetic mechanism of various chondropathies. Another innovation is that we integrated TWAS results with different mRNA expression profiles of three disorders of chondropathies to find the common genes. The results of these common genes could be more reliable because they are not only based on the TWAS results, but also verify the TWAS results according to different mRNA profiles. Additionally, the aim of this study is to genome-wide scan candidate genes for chondropathies, through integrating TWAS and mRNA expression profile data. To validate the accuracy of integrative analysis results, two knee OA associated candidate genes were selected for IHC validation. Further functional studies are needed to confirm our findings and clarify the potential mechanism of identified candidate genes involved in the development of chondropathies.

\section{Conclusions}

In conclusion, we conducted an integrative analysis of TWAS and mRNA expression profiles and identified multiple knee OA-, CT-, and SDH-related candidate genes and GO terms. Our findings may provide a novel insight into the pathogenesis of chondropathies.

\section{Additional files}

Additional file 1: Table S1. The characteristic of the mRNA expression study samples. (DOCX $14 \mathrm{~kb}$ )

Additional file 2: TableS2. The basic characteristics of study samples for IHC. (DOCX $13 \mathrm{~kb}$ )

Additional file 3: Table S3. TWAS identified significant genes in MS for chondropathies. (DOCX $29 \mathrm{~kb}$ )

Additional file 4: Table S4. TWAS identified significant genes in YBL for chondropathies. (DOCX $34 \mathrm{~kb}$ )

Additional file 5: Table S5. Interative analyses identified candidate genes for knee OA. (DOCX 26 kb)

Additional file 6: Table S6. Interative analyses identified causal genes for cartilage tumor. (DOCX $16 \mathrm{~kb}$ )

Additional file 7: Table S7. Interative analyses identified causal genes for spinal disc herniation. (DOCX $15 \mathrm{~kb}$ )

Additional file 8: Table S8. GO enrichment analyses results for knee OA. (DOCX $23 \mathrm{~kb})$

\section{Abbreviations}

ABCA1: ATP-binding-cassette transporter A1; ApoA1: Apoliproprotein A1; $C T$ : Cartilage tumor; FC: Fold change; FUMA: Functional mapping and annotation; GO: Gene ontology; GWAS: Genome-wide association studies; HNSCC: Head and neck squamous cell carcinoma; HRP: Horseradish peroxidase; IL-1 $\beta$ : Interleukin 1 $\beta$; IVD: Intervertebral disc; LIMMA: Linear models for microarray; LXR a: Liver X receptor alpha; LXR $\beta$ : Liver $X$ receptor beta; MS: Muscle skeleton; NF-kB: Nuclear factor-kB; OA: Osteoarthritis; qRTPCR: Quantitative reverse transcription polymerase chain reaction: ROS: Reactive oxygen species; RT: Room temperature; SDH: Spinal disc herniation; TNF-a: Tumor necrosis factor a; TWAS: Transcriptome-wide association study; YBL: Peripheral blood

\section{Acknowledgements}

This work was supported by the National Natural Scientific Foundation of China [81673112]; the Key projects of international cooperation among governments in scientific and technological innovation [2016YFE0119100]; the Natural Science Basic Research Plan in Shaanxi Province of China [2017JZ024]; and the Fundamental Research Funds for the Central Universities.

\section{Authors' contributions}

FZ designed this study. XG gave the conception and some critical suggestions to this study. FZ, PL, YJN, YW, BLC, MM, SQC, LZ, and SW conducted the experiments and data analysis. PL and YJN convinced the project and wrote the manuscript. All authors read and approved the final manuscript.

\section{Funding}

This work was supported by the National Natural Scientific Foundation of China [81673112]; the Key projects of international cooperation among governments in scientific and technological innovation [2016YFE0119100]; the Natural Science Basic Research Plan in Shaanxi Province of China [2017JZ024]; and the Fundamental Research Funds for the Central Universities.

\section{Availability of data and materials}

The datasets used and/or analyzed during the current study are available from the corresponding author on reasonable request.

Ethics approval and consent to participate

This study was approved by the Human Ethics Committees of Xi'an Jiaotong University (Project identification code: 2015-237). Informed consent documents were signed by all participants.

\section{Consent for publication}

Not applicable.

\section{Competing interests}

The authors declare that they have no competing interests.

Received: 13 April 2019 Accepted: 16 August 2019

Published online: 27 August 2019

References

1. Krishnan Y, Grodzinsky AJ. Cartilage diseases. Matrix Biol. 2018;71-72:51-69.

2. Johnson VL, Hunter DJ. The epidemiology of osteoarthritis. Best Pract Res Clin Rheumatol. 2014;28(1):5-15.

3. Al Nezari NH, Schneiders AG, Hendrick PA. Neurological examination of the peripheral nervous system to diagnose lumbar spinal disc herniation with suspected radiculopathy: a systematic review and meta-analysis. Spine J. 2013;13(6):657-74

4. Valdes AM, Evangelou E, Kerkhof HJ, Tamm A, Doherty SA, Kisand K, Tamm A, Kerna I, Uitterlinden A, Hofman A, et al. The GDF5 rs143383 polymorphism is associated with osteoarthritis of the knee with genome -wide statistical significance. Ann Rheum Dis. 2011;70(5):873-5.

5. Ho L, Stojanovski A, Whetstone H, Wei QX, Mau E, Wunder JS, Alman B. Gli2 and p53 cooperate to regulate IGFBP-3- mediated chondrocyte apoptosis in the progression from benign to malignant cartilage tumors. Cancer Cell. 2009;16(2):126-36

6. Ala-Kokko L. Genetic risk factors for lumbar disc disease. Ann Med. 2002;34(1):42-7.

7. Makris EA, Gomoll AH, Malizos KN, Hu JC, Athanasiou KA. Repair and tissue engineering techniques for articular cartilage. Nat Rev Rheumatol. 2015;11(1):21-34.

8. Dye SF. The pathophysiology of patellofemoral pain: a tissue homeostasis perspective. Clin Orthop Relat Res. 2005:436:100-10.

9. Grenier S, Bhargava MM, Torzilli PA. An in vitro model for the pathological degradation of articular cartilage in osteoarthritis. J Biomech. 2014;47(3):645-52.

10. van Vulpen LF, Schutgens RE, Coeleveld K, Alsema EC, Roosendaal G, Mastbergen SC, Lafeber FP. IL-1beta, in contrast to TNFalpha, is pivotal in 
blood-induced cartilage damage and is a potential target for therapy. Blood. 2015;126(19):2239-46.

11. Yau MS, Yerges-Armstrong LM, Liu Y, Lewis CE, Duggan DJ, Renner JB, Torner J, Felson DT, McCulloch CE, Kwoh CK, et al. Genome-wide association study of radiographic knee osteoarthritis in North American Caucasians. Arthritis Rheumatol. 2017:69(2):343-51.

12. Lu Y, Beeghly-Fadiel A, Wu L, Guo X, Li B, Schildkraut JM, Im HK, Chen YA, Permuth JB, Reid BM, et al. A transcriptome-wide association study among 97,898 women to identify candidate susceptibility genes for epithelial ovarian cancer risk. Cancer Res. 2018;78(18):5419-30.

13. Gusev A, Ko A, Shi H, Bhatia G, Chung W, Penninx BW, Jansen R, de Geus EJ, Boomsma DI, Wright FA, et al. Integrative approaches for large-scale transcriptome-wide association studies. Nat Genet. 2016;48(3):245-52.

14. Bycroft C, Freeman C, Petkova D, Band G, Elliott LT, Sharp K, Motyer A, Vukcevic D, Delaneau O, O'Connell J, et al. The UK Biobank resource with deep phenotyping and genomic data. Nature. 2018;562(7726):203-9.

15. Canela-Xandri O, Rawlik K, Tenesa A. An atlas of genetic associations in UK Biobank. Nat Genet. 2018;50(11):1593-9.

16. Fu M, Huang G, Zhang Z, Liu J, Zhang Z, Huang Z, Yu B, Meng F. Expression profile of long noncoding RNAs in cartilage from knee osteoarthritis patients. Osteoarthr Cartil. 2015;23(3):423-32.

17. Pansuriya TC, Oosting J, Krenacs T, Taminiau AH, Verdegaal SH, Sangiorgi L, Sciot R, Hogendoorn PC, Szuhai K, Bovee JV. Genome-wide analysis of Ollier disease: is it all in the genes? Orphanet J Rare Dis. 2011;6:2.

18. Zhang YG, Guo X, Sun Z, Jia G, Xu P, Wang S. Gene expression profiles of disc tissues and peripheral blood mononuclear cells from patients with degenerative discs. J Bone Miner Metab. 2010;28(2):209-19.

19. Smyth GK. Linear models and empirical bayes methods for assessing differential expression in microarray experiments. Stat Appl Genet Mol Biol. 2004;3:Article3.

20. Watanabe $K$, Taskesen E, van Bochoven A, Posthuma D. Functional mapping and annotation of genetic associations with FUMA. Nat Commun. 2017;8(1):1826.

21. Takata A, Otsuka M, Yoshikawa T, Kishikawa T, Hikiba Y, Obi S, Goto T, Kang YJ, Maeda S, Yoshida H, et al. MicroRNA-140 acts as a liver tumor suppressor by controlling NF-kappaB activity by directly targeting DNA methyltransferase 1 (Dnmt1) expression. Hepatology. 2013;57(1):162-70.

22. Nakatomi $C$, Nakatomi M, Matsubara T, Komori T, Doi-Inoue T, Ishimaru N, Weih F, Iwamoto T, Matsuda M, Kokabu S, et al. Constitutive activation of the alternative NF-kappaB pathway disturbs endochondral ossification. Bone. 2019:121:29-41.

23. Xing J, Nan X, Cui Q, Ma W, Zhao H. Nop-7-associated 2 (NSA2) is required for ribosome biogenesis and protein synthesis. Biochem Biophys Res Commun. 2018;505(1):249-54

24. Shahni R, Czajka A, Mankoo BS, Guvenel AK, King AJ, Malik AN. Nop-7 -associated 2 (NSA2), a candidate gene for diabetic nephropathy, is involved in the TGFbeta1 pathway. Int J Biochem Cell Biol. 2013;45(3):626-35.

25. He L, Zhou X, Qu C, Tang Y, Zhang Q, Hong J. Serglycin (SRGN) overexpression predicts poor prognosis in hepatocellular carcinoma patients. Med Oncol. 2013;30(4):707.

26. Korpetinou A, Papachristou DJ, Lampropoulou A, Bouris P, Labropoulou VT, Noulas A, Karamanos NK, Theocharis AD. Increased expression of serglycin in specific carcinomas and aggressive cancer cell lines. Biomed Res Int. 2015;2015:690721.

27. Jiang $H$, Hu H, Tong $X$, Jiang $Q$, Zhu H, Zhang S. Calcium-binding protein S100P and cancer: mechanisms and clinical relevance. J Cancer Res Clin Oncol. 2012;138(1):1-9.

28. Yang R, Stocker S, Schott S, Heil J, Marme F, Cuk K, Chen B, Golatta M, Zhou Y, Sutter C, et al. The association between breast cancer and S100P methylation in peripheral blood by multicenter case-control studies. Carcinogenesis. 2017;38(3):312-20

29. Piltti J, Bygdell J, Qu C, Lammi MJ. Effects of long-term low oxygen tension in human chondrosarcoma cells. J Cell Biochem. 2018;119(2):2320-32.

30. Qu J, Lu D, Guo H, Miao W, Wu G, Zhou M. PFKFB3 modulates glycolytic metabolism and alleviates endoplasmic reticulum stress in human osteoarthritis cartilage. Clin Exp Pharmacol Physiol. 2016;43(3):312-8.

31. Bartrons R, Rodriguez-Garcia A, Simon-Molas H, Castano E, Manzano A, Navarro-Sabate A. The potential utility of PFKFB3 as a therapeutic target. Expert Opin Ther Targets. 2018;22(8):659-74.

32. Hartmann TB, Mattern E, Wiedemann N, van Doorn R, Willemze R, Niikura T, Hildenbrand R, Schadendorf D, Eichmuller SB. Identification of selectively expressed genes and antigens in CTCL. Exp Dermatol. 2008;17(4):324-34.
33. Zhu MH, Ji SL, Zhang CY, Cui L, Xiong L, Zheng HL. DNA microarray reveals ZNF195 and SBF1 are potential biomarkers for gemcitabine sensitivity in head and neck squamous cell carcinoma cell lines. Int J Clin Exp Pathol. 2014;7(4):1514-23.

34. Blanco FJ, Rego I, Ruiz-Romero C. The role of mitochondria in osteoarthritis. Nat Rev Rheumatol. 2011:7(3):161-9.

35. Lopez de Figueroa P, Lotz MK, Blanco FJ, Carames B. Autophagy activation and protection from mitochondrial dysfunction in human chondrocytes. Arthritis Rheumatol. 2015;67(4):966-76.

36. Tang $Q$, Zheng G, Feng Z, Chen Y, Lou Y, Wang C, Zhang X, Zhang Y, Xu H, Shang $P$, et al. Trehalose ameliorates oxidative stress-mediated mitochondrial dysfunction and ER stress via selective autophagy stimulation and autophagic flux restoration in osteoarthritis development. Cell Death Dis. 2017;8(10):e3081.

37. Thijssen E, van Caam A, van der Kraan PM. Obesity and osteoarthritis, more than just wear and tear: pivotal roles for inflamed adipose tissue and dyslipidaemia in obesity-induced osteoarthritis. Rheumatology. 2015;54(4): 588-600.

38. Gkretsi V, Simopoulou T, Tsezou A. Lipid metabolism and osteoarthritis: lessons from atherosclerosis. Prog Lipid Res. 2011;50(2):133-40.

39. Tsezou A, lliopoulos D, Malizos KN, Simopoulou T. Impaired expression of genes regulating cholesterol efflux in human osteoarthritic chondrocytes. J Orthop Res. 2010;28(8):1033-9.

40. Byun S, Sinskey YL, Lu YC, Frank EH, Grodzinsky AJ. Transport and binding of tumor necrosis factor-alpha in articular cartilage depend on its quaternary structure. Arch Biochem Biophys. 2013;540(1-2):1-8.

41. Kapoor M, Martel-Pelletier J, Lajeunesse D, Pelletier JP, Fahmi H. Role of proinflammatory cytokines in the pathophysiology of osteoarthritis. Nat Rev Rheumatol. 2011;7(1):33-42.

42. Spinazzi M, Sghirlanzoni A, Salviati L, Angelini C. Impaired copper and iron metabolism in blood cells and muscles of patients affected by copper deficiency myeloneuropathy. Neuropathol Appl Neurobiol. 2014; 40(7):888-98.

\section{Publisher's Note}

Springer Nature remains neutral with regard to jurisdictional claims in published maps and institutional affiliations.

\section{Ready to submit your research? Choose BMC and benefit from:}

- fast, convenient online submission

- thorough peer review by experienced researchers in your field

- rapid publication on acceptance

- support for research data, including large and complex data types

- gold Open Access which fosters wider collaboration and increased citations

- maximum visibility for your research: over $100 \mathrm{M}$ website views per year

At $\mathrm{BMC}$, research is always in progress.

Learn more biomedcentral.com/submission 